\title{
Mild hypothermia pretreatment protects against liver ischemia reperfusion injury via the PI3K/AKT/FOXO3a pathway
}

\author{
QI XIAO ${ }^{1}$, QIFA YE $^{1,2}$, WEI WANG $^{1}$, JIANSHENG XIAO $^{3}$, BIQI FU ${ }^{4}$, ZHIPING XIA $^{2}$, \\ XINGJIAN ZHANG ${ }^{2}$, ZHONGZHONG LIU ${ }^{2}$ and XIANPENG ZENG ${ }^{2}$
}

\begin{abstract}
${ }^{1}$ Department of Transplant Surgery, The Third Xiangya Hospital of Central South University, Central South University, Changsha, Hunan 410013; ${ }^{2}$ Hubei Key Laboratory of Medical Technology on Transplantation, Zhongnan Hospital of Wuhan University, Institute of Hepatobiliary Diseases of Wuhan University, Transplant Center of Wuhan University, Wuhan, Hubei 430071; Departments of ${ }^{3}$ Transplant Surgery and ${ }^{4}$ Rheumatology and Immunology, The First Affiliated Hospital of Nanchang University, Nanchang, Jiangxi 330006, P.R. China
\end{abstract}

Received January 6, 2017; Accepted July 26, 2017

DOI: $10.3892 / \mathrm{mmr} .2017 .7501$

\begin{abstract}
Mild hypothermia is known to protect against ischemia and reperfusion (IR) injury. The exact mechanisms of the protection are not fully understood. Forkhead box O3 (FOXO3a) has been defined as a critical mediator in cellular processes, including oxidative stress, apoptosis, inflammation, cell death and DNA repair; however, the protection function in mild hypothermia has not been reported previously. The current study was designed to investigate the function of phosphoinositide 3-kinase (PI3K)/protein kinase B (AKT)/FOXO3a pathway in pretreatment with mild hypothermia during IR injury. Additionally, PI3K/AKT/FOXO3a signaling was inhibited using Ly294002 and the effect on the protective function of mild hypothermia pretreatment was evaluated. Furthermore, the apoptotic and inflammatory response induced by the IR injury was evaluated. Liver IR injury induced a significant increase in the level of apoptosis and inflammatory responses. However, pretreatment with mild hypothermia increased phospho (p)-AKT and p-FOXO3a following IR injury, and significantly reduced apoptosis and inflammatory cytokines release. However, inhibiting p-AKT and p-FOXO3a using Ly294002 suppressed the liver protection produced by mild hypothermia. In conclusion, these findings indicated that mild
\end{abstract}

Correspondence to: Dr Qifa Ye, Department of Transplant Surgery, The Third Xiangya Hospital of Central South University, Central South University, 172 Tongzipo Road, Changsha, Hunan 410013, P.R. China

E-mail: yqf_china@163.com

Dr Jiansheng Xiao, Department of Transplant Surgery, The First Affiliated Hospital of Nanchang University, 17 Yongwai Zhengjie Street, Nanchang, Jiangxi 330006, P.R. China

E-mail: super_xiaoj@163.com

Key words: mild hypothermia pretreatment, liver ischemia reperfusion, phosphoinositide 3-kinase/protein kinase B/forkhead box $\mathrm{O} 3$ pathway, apoptosis, inflammatory hypothermia pretreatment exhibited liver protective effects against IR injury associated with suppressing inflammatory cytokine release and apoptosis via the PI3K/AKT/FOXO3a pathway.

\section{Introduction}

Liver injury induced by hepatic ischemia/reperfusion (IR) is a major cause of hepatic failure following various forms of circulatory shock, severe trauma, liver resection, orthotropic liver transplantation or other surgical procedures where blood supply to the liver is temporarily blocked (1). The mechanism of IR injury of the liver is complicated and interactive. During the phase of IR, hepatocyte damage occurs through lipid peroxidation, release of reactive oxygen species, activation of signal transduction cascades and production of various inflammatory mediators (2). Subsequently, cell death programs are activated, including apoptosis, autophagy-associated cell death and necrosis (3).

Several strategies, such as ischemic preconditioning, remote ischemic preconditioning or pharmacological preconditioning, that may protect the liver during IR injury have been investigated (4). Among these strategies, mild hypothermia has received increasing attention. The modern clinical use of hypothermia was initiated in 1950 by Bigelow et al (5) when the neuroprotective effects were demonstrated during cardiac surgery. For many years, therapeutic hypothermia has been applied to improve the survival rate and neurological outcome of patients following cardiac arrest. The beneficial effects of therapeutic hypothermia include conservation of hepatic metabolism, and reduction in the inflammatory response and apoptosis during ischemia $(6,7)$. However, there is a lack of consensus on the most appropriate target temperature. Experimental studies $(8,9)$ in recent years have suggested that mild hypothermia $\left(32-34^{\circ} \mathrm{C}\right)$ exert more effective protection against warm IR injury of the liver. Previous studies have focused on the investigation of therapeutic hypothermia at $32-34^{\circ} \mathrm{C}$. However, in selective cases the application of mild hypothermia pretreatment may outweigh the risks, whereas fewer studies were conducted on it and the mechanisms by 
which mild hypothermia pretreatment protects liver against I/R injury are not fully understood.

Previous studies $(10,11)$ demonstrate that the phosphatidylinositol-3-kinase (PI3K)/protein kinase B (AKT) pathway has an important role in protection against hepatic IR injury in rats. Activated AKT inhibits apoptosis by phosphorylating a large number of downstream proteins (12). Several genetic and biochemical studies have reported that the forkhead box $\mathrm{O}$ (FOXO) family is a key downstream target of the PI3K/AKT pathway $(13,14)$. FOXO3a is a member of the FOXO family of transcription factors and is expressed in the liver, pancreas, spleen, brain and other tissues (15). FOXO3a induces expression of target genes involved in crucial cellular processes, including oxidative stress, glucose metabolism, inflammation response and apoptosis (16). There are multiple mechanisms that post-translationally regulate transcriptional activity of FOXO3a transcription factors (17). Notably, phospho (p)-AKT regulates phosphorylation of FOXO3a, which increases translocation of p-FOXO3a from the nucleus to the cytosol, inhibiting the transcriptional activity of FOXO3a. In turn, dephosphorylation of FOXO3a increases its nuclear activity, which enhances target gene expression (16). However, the majority of previous studies assessed p-AKT alone without focusing on its substrates, such as FOXO3a. The activation of FOXO3a may have an important role in IR injury.

Based on the above reports, it is well established that mild hypothermia possesses potent anti-apoptosis activity and inhibits the inflammatory response. The aim of the present study was to determine whether the use of mild hypothermia pretreatment protects the liver, reducing ischemia-reperfusion injury. Furthermore, whether the activation of PI3K/AKT/FOXO3a pathways contributed to the induction of liver protection in this type of pretreatment via anti-apoptosis and inhibited the inflammatory response were investigated.

\section{Materials and methods}

Animals. Male Sprague-Dawley rats ( $\mathrm{n}=48 ; 3$ months old; weight 250-280 g) were purchased from Hubei Provincial Center for Disease Control and Prevention (Wuhan, China), and then housed in the animal center of Zhongnan Hospital of Wuhan University (Wuhan, China). Rats were housed at $22 \pm 2{ }^{\circ} \mathrm{C}$ with a 12 -h light/dark cycle, $60 \pm 5 \%$ humidity and given food and water ad libitum. They were allowed to adapt to the environment for 3 days prior to the experiment. All the rats were randomly divided into 6 groups, each group had 8 rats: i) Normal (N) group; ii) mild hypothermic pretreatment (MH) group; iii) sham (S) group; iv) normothermic pretreatment + IR (NH + IR) group; v) mild hypothermic pretreatment + IR (MH + IR) group; vi) Ly294002 + mild hypothermic pretreatment + IR ( $\mathrm{Ly}+\mathrm{MH}+\mathrm{IR})$ group. All animals were fasted $12 \mathrm{~h}$ before the experiment, with free access to water. All experimental procedures were approved by the Ethics Review Committee of Zhongnan Hospital of Wuhan University and every effort was taken to minimize pain and discomfort of rats.

Model for liver IR. The rats in the $\mathrm{MH}, \mathrm{MH}+\mathrm{IR}$, and $\mathrm{Ly}+\mathrm{MH}+\mathrm{IR}$ groups were cooled until their rectal temperatures were reduced to $32.2 \pm 0.5^{\circ} \mathrm{C}$. Mild hypothermia pretreatment was maintained for $2 \mathrm{~h}$ by maintaining the rat with an icebox and a heat lamp. The rectal temperatures of the rats in the $\mathrm{N}, \mathrm{S}$, and $\mathrm{N}+\mathrm{IR}$ groups were also maintained at $37 \pm 0.5^{\circ} \mathrm{C}$ for $2 \mathrm{~h}$.

Pre-treatment with PI3K inhibitor (Ly294002; $1.5 \mathrm{mg} / \mathrm{kg}$ ) and vehicle [10\% dimethyl sulfoxide (DMSO) and 90\% PBS. was given intraperitoneally (i.p.) $30 \mathrm{~min}$ prior to the mild hypothermic pretreatment in the $\mathrm{Ly}+\mathrm{MH}+\mathrm{IR}$ group. The other rats in the $\mathrm{S}, \mathrm{NH}+\mathrm{IR}$, and $\mathrm{MH}+\mathrm{IR}$ groups were injected with an equivalent volume of vehicle (10\% DMSO and $90 \%$ PBS).

The rats were anesthetized with chloral hydrate $(40 \mathrm{mg} / \mathrm{kg}$, i.p.) during surgery. Then a midline incision was made to expose the liver and free the perhepatic ligament, injected $100 \mathrm{IU} / \mathrm{kg}$ heparin for systemic anti-coagulation through a penile vein. The artery and portal venous blood supply to the left/middle liver lobes were blocked with an atraumatic clip for $60 \mathrm{~min}$. The abdomen was closed temporarily until re-opened, and the clamp was removed to induce reperfusion. The rectal temperature of the rat was maintained at $37 \pm 0.5^{\circ} \mathrm{C}$ in the entire surgical procedure. After $6 \mathrm{~h}$ of reperfusion, the rats were reanesthetized and sacrificed to collect hepatic tissues and blood samples immediately. The sham group rats underwent the same surgical procedure without vascular occlusion. The $\mathrm{N}$ group and $\mathrm{MH}$ group did not receive surgery.

Serum alanine aminotransferase ALT levels. Serum SALT level, an indicator of hepatocellular injury, was measured in blood samples using an automated chemical analyzer.

Liver histology. Ringer $\left(0-4^{\circ} \mathrm{C}\right.$, heparinized, $\left.20 \mathrm{ml}\right)$ was used to flush the blood from the liver via the abdominal aorta, then fixed in $10 \%$ formalin and embedded in paraffin. Paraffin sections were cut into $5 \mu \mathrm{m}$ and stained with hematoxylin and eosin (H\&E) according to standard protocols (18). Stained tissue slices were observed with a bright field microscope by a pathologist. The severity of liver IR injury was graded blindly using the criteria of Suzuki et al (19) on a scale from 0-4.

Myeloperoxidase (MPO) assay. The presence of MPO was used as an index of neutrophil accumulation in the liver and was obtained from Nanjin Jianchen Biotechnology, (Nanjin, China; http://www.njjcbio.com). MPO activity was measured according to the manufacturer's protocols.

Serum cytokines. Interleukin-1 $\beta$ (IL-1 $\beta$ ) and tumor necrosis factor- $\alpha(\mathrm{TNF}-\alpha)$ and IL- 6 were quantified from serum by ELISA and were obtained from Cloud-Clone Corp. (Katy, TX, USA).

Terminal deoxynucleotidyl transferase (TdT) dUTP nick-end labelling (TUNEL) detection. Sections were permeabilized in xylene, dehydrated in graded ethanol series to water, incubation with $3 \%$ hydrogen peroxide for $30 \mathrm{~min}$, then treated with proteinase $\mathrm{K}(5 \mathrm{mg} / \mathrm{ml})$ for $5 \mathrm{~min}$ at room temperature. Subsequently, the sections were applied with TdT reaction buffer (Roche Diagnostics GmbH, Mannheim, Germany) for $1 \mathrm{~h}$ at $37^{\circ} \mathrm{C}$. Following washing in PBS, the sections were incubated with converter-peroxidase and detected with diaminobenzidine. 


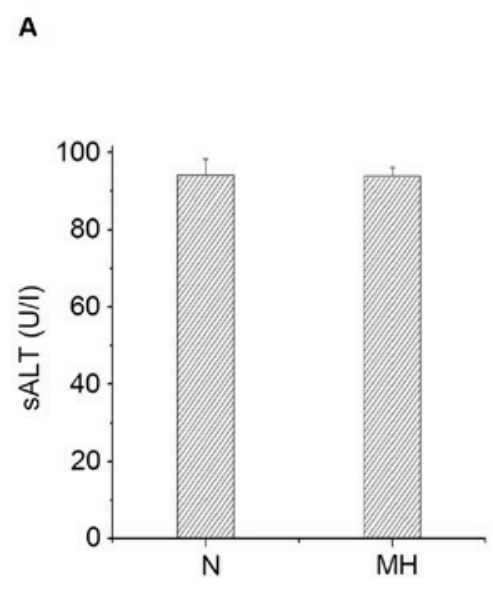

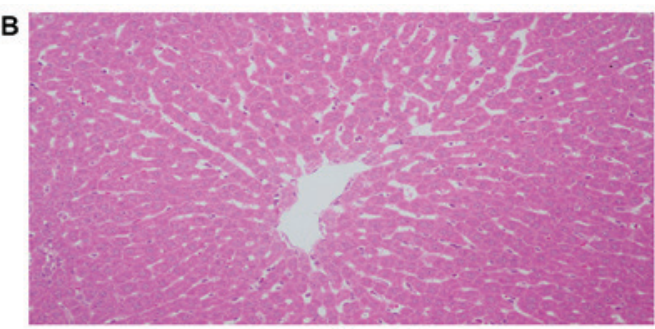

N

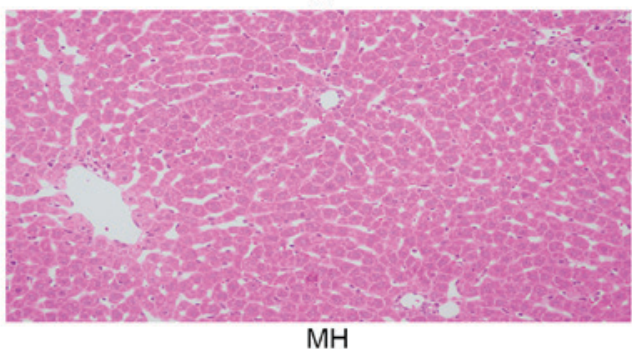

Figure 1. Mild hypothermia did not damage hepatocellular function. (A) Hepatocellular function evaluated by sALT (U/1) levels. Values are expressed as the mean \pm standard deviation ( $\mathrm{n}=8$ /group). $\mathrm{P}>0.05, \mathrm{~N}$ group vs. $\mathrm{MH}$ group. (B) Representative liver histology of ischemic liver lobes, hematoxylin and eosin staining. Sinusoidal congestion and vacuolization, and necrosis were not observed in the N and MH groups (magnification, $\mathrm{x} 200$, $\mathrm{n}=8 / \mathrm{group}$ ). sALT, serum alanine aminotransferase; $\mathrm{N}$, normal; $\mathrm{MH}$, mild hypothermic pretreatment.

Reverse transcription-quantitative polymerase chain reaction $(R T-q P C R)$. Total RNA was obtained from liver tissue with TRIzol reagent (Invitrogen; Thermo Fisher Scientific, Inc., Waltham,MA, USA) according to the manufacturer's protocols, and cDNA for RT-qPCR was synthesized using oligo $\mathrm{d}(\mathrm{T})$ and a Superscript III RT kit (Thermo Fisher Scientific, Inc.). qPCR was conducted using a Quantitative SYBR-Green RT-PCR kit (Takara Bio, Inc., Otsu, Japan) and an Applied Biosystems 7500 system (Thermo Fisher Scientific, Inc.). All reactions were conducted in a $20 \mu \mathrm{l}$ reaction volume in triplicate. The relative expression levels for a target gene were normalized to $\beta$-actin. Specificity was verified by melting curve analysis and agarose gel electrophoresis. FOXO3a, nuclear factor (NF) $-\kappa B$ and $\beta$-actin were obtained from Wuhan GuGe Biotechnology, (Wuhan China). Primers were as follows: FOXO3a sense, 5'-AACAGTACCGTGTTCGGACC-3' and anti-sense, 5'-AGT GTCTGGTTGCCGTAGTG-3'; NF-кB sense, 5'-GCTCCT TTTCTCAAGCCGATGT-3' and anti-sense, 5'-CGTAGGTCC TTTTGCGTTTTTC-3'; and $\beta$-actin sense, 5'-GTTACAGGA AGTCCCTCACCC-3' and anti-sense, 5'-CAGACCTGGGCC ATTCAGAAA-3'. Data were analyzed using the comparative $\mathrm{C}_{\mathrm{q}}\left(2^{-\Delta \Delta \mathrm{Cq}}\right)(20)$.

Western blotting. Total protein was obtained from liver tissue with a triple detergent RIPA buffer (Solarbio, Wuhan, China) containing a protease inhibitor cocktail and nuclear protein was obtained from liver tissue with nuclear and cytoplasmic protein extraction kit (Beyotime Institute of Biotechnology, Beijing, China) according to the manufacturer's protocols. Approximately $40 \mu \mathrm{g}$ of proteins were separated by $10 \%$ SDS-PAGE and transferred onto polyvinylidene membranes. Membranes were blocked with 5\% skimmed milk in PBS at room temperature for $2 \mathrm{~h}$ and then incubated with the corresponding primary antibodies: p-AKT (ser473; ab81283; 1:1,000), p-FOXO3a (ser253; ab47285; 1:1,000), FOXO3a (ab109629; 1:1,000), NF-кB (ab16502; 1:1,000), Bcl-2 associated $\mathrm{X}$ apoptosis regulator (Bax; ab32503; 1:1,000), Bcl-2 apoptosis regulator (Bcl-2; ab59348; 1:1,000; all from Abcam, Cambridge, UK), p-NF- $\mathrm{B}$ inhibitor $\alpha$ (IKB $\alpha$; ser32; 2859; 1:1,000; Cellular Technology Ltd., Cleveland, OH, USA) at $4^{\circ} \mathrm{C}$ overnight. Membranes were then washed with Tris-buffered saline-Tween repeatedly and incubated with secondary antibodies (SE132; 1:3,000; Beijing Solarbio Science and Technology Co., Ltd., Beijing, China) at room temperature for 45 min. Enhanced chemiluminescence (Wuhan Sanying Biotechnology, Wuhan, China) was used to visualize the immunoreactive bands. Signal intensities of all protein bands were assessed by the Quantity One software version 4.62 (Bio-Rad Laboratories, Inc., Hercules, CA, USA). Anti-rat $\beta$-actin (K006153P; 1:3,000; Wuhan GuGe Biotechnology, Wuhan, China) and TATA-box binding protein (22006-1-AP, 1:3,000; Wuhan Sanying Biotechnology) were used as internal controls.

Statistical analysis. All data are expressed as the mean \pm standard deviation ( $\mathrm{n}=8 /$ group). Comparison between two groups were performed using Student's t-test, while the differences among $\geq 3$ groups were analyzed by one-way analysis of variance for post hoc multiple comparisons. $\mathrm{P}<0.05$ was considered to indicate a statistically significant difference.

\section{Results}

Mild hypothermia pretreatment does not damage hepatocellular function. The hepatocellular function was analyzed in rat livers subjected to mild hypothermia pretreatment without any operation. As presented in Fig. 1A, there was no difference in the sALT level between the $\mathrm{N}$ and $\mathrm{MH}$ group (94.23 \pm 4.18 vs. 93.85 $\pm 2.38 \mathrm{IU} / \mathrm{l})$. These data were supported by H\&E staining (Fig. 1B). The MH group and $\mathrm{N}$ group did not exhibit sinusoidal congestion, vacuolization or necrosis. These data indicated that only giving mild hypothermia pretreatment would not damage hepatocellular function, thus, the effects of mild hypothermia pretreatment only were not examined further. 
A

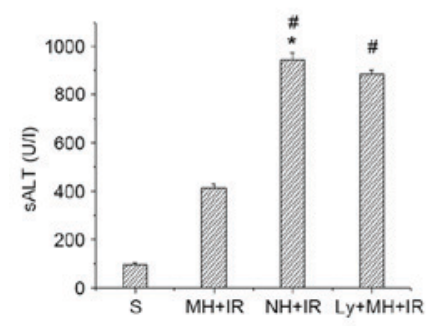

C

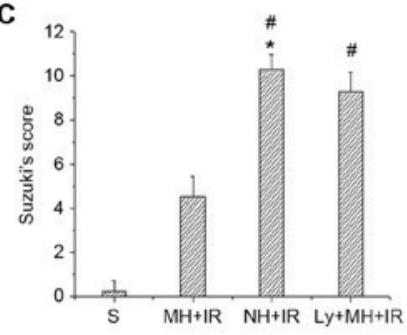

B

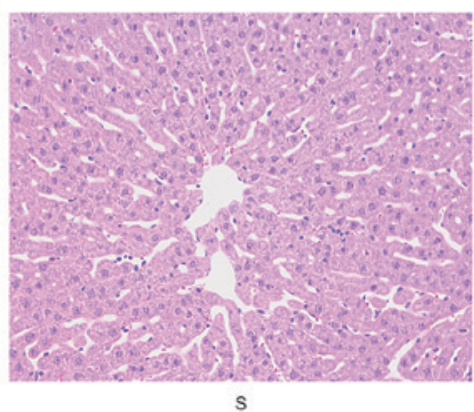

s

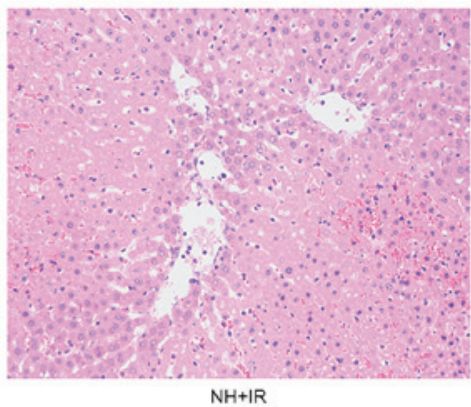

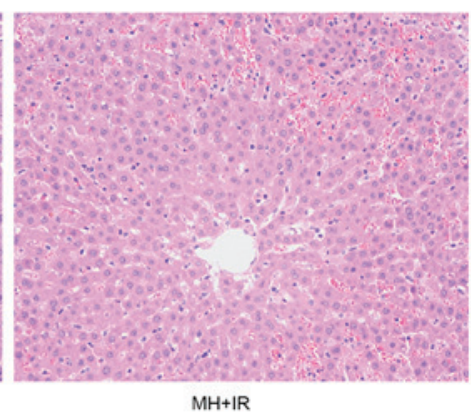

$\mathrm{MH}+\mathrm{IR}$

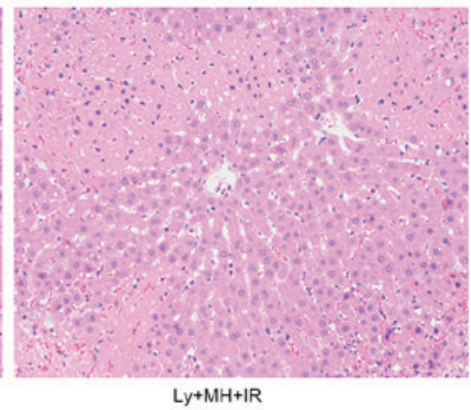

Figure 2. Mild hypothermia promotes anti-apoptotic function and reduces hepatocellular apoptosis in liver IR injury (6 h reperfusion after $60 \mathrm{~min}$ warm ischemia). (A) Hepatocellular function evaluated by SALT (U/I) levels. (B) Representative liver histology of ischemic liver lobes (H\&E staining; magnification, $\mathrm{x} 200$ ). (C) Suzuki's histological grading of liver IR injury. Values re-expressed as the mean \pm standard deviation ( $\mathrm{n}=8 /$ group). ${ }^{*} \mathrm{P}<0.05$ vs. $\mathrm{S}$ group, ${ }^{\#} \mathrm{P}<0.05$ vs. MH + IR group. sALT, serum alanine aminotransferase; S, sham; MH, mild hypothermia pretreatment; IR, ischemia/reperfusion; NH, normothermic pretreatment; Ly, Ly294002.

Mild hypothermia pretreatment protects the liver against IRI. The hepatocellular function was analyzed in rat livers subjected to $60 \mathrm{~min}$ of warm ischemia followed by $6 \mathrm{~h}$ reperfusion. As presented in Fig. 2A, sALT levels in the MH + IR group was decreased compared with the NH + IR group $(414.65 \pm 12.74$ vs. $943.52 \pm 32.3$ IU/1). These data were supported by Suzuki's histological grading of liver IRI (Fig. 2B and C). Indeed, mild hypothermia pretreatment resulted in minimal liver sinusoidal congestion, vacuolization and necrosis (score, 4.5 \pm 0.93 ; Fig. 2B and C). By contrast, untreated livers (NH + IR) exhibited severe edema and extensive hepatocellular necrosis (score, 10.25 \pm 0.7 ; Fig. 2B and C). However, the protective effect of mild hypothermia pretreatment was blocked when rats were pre-treated with Ly294002 (885.2 \pm 14.51 IU/1, Fig. 2A; and score, 9.25 \pm 0.89 , Fig. 2B and C).

Mild hypothermia pretreatment activates PI3K/AKT/FOXO3a signaling in liver IRI. To further investigate the role of $\mathrm{PI} 3 \mathrm{~K} / \mathrm{AKT} / \mathrm{FOXO} 3 \mathrm{a}$ signaling in liver IRI protection by mild hypothermia pretreatment, the expression levels of FOXO3a mRNA and p-AKT, p-FOXO3a and nuclear FOXO3a protein were determined. When compared with the $\mathrm{NH}+\mathrm{IR}$ group and $\mathrm{Ly}+\mathrm{MH}+\mathrm{IR}$, mild hypothermia pretreatment increased p-AKT and p-FOXO3a protein (Fig. 3A-C). Notably, the mRNA and nuclear FOXO3a protein expression were significantly reduced in the MH+IR group compared with the $\mathrm{NH}+\mathrm{IR}$ group and Ly + MH + IR group (Fig. 3A, D and E).

Mild hypothermia pretreatment promotes anti-apoptotic function and reduces hepatocellular apoptosis in liver IR injury. In order to study the protective effect of mild hypothermia pretreatment against cell death induced by IR, the expression of anti-apoptotic (Bcl-2) and pro-apoptotic (Bax) proteins was determined. Indeed, the Bcl-2 protein expression was MH + IR group markedly increased in the ischemic liver compared with the $\mathrm{NH}+\mathrm{IR}$ or $\mathrm{Ly}+\mathrm{MH}+\mathrm{IR}$ group (Fig. 3A and F). Furthermore, the expression of Bax was lower in the MH + IR group compared with the $\mathrm{NH}+\mathrm{IR}$ or $\mathrm{Ly}+\mathrm{MH}+\mathrm{IR}$ group (Fig. 3A and G). Hepatocellular apoptosis was also determined using TUNEL staining. Mild hypothermia pretreatment decreased the number of TUNEL-positive cells compared with $\mathrm{NH}+$ IR group or Ly + MH + IR (Fig. 4A and B).

Mild hypothermia pretreatment inhibits the release of proinflammatory mediators in liver IRI. IR activates a redox sensitive transcription factor $\mathrm{NF}-\kappa \mathrm{B}$ and the activation of $\mathrm{NF}-\kappa \mathrm{B}$ induces the expression of proinflammatory mediators. Whether mild hypothermia pretreatment contributed to inhibit this was investigated. As presented in Fig. 3A and I, when compared with the NH + IR and Ly + MH + IR groups, mild hypothermia pretreatment reduced $\mathrm{p}-\mathrm{IKB} \alpha$ protein, and decreased protein and mRNA expression of NF- $\kappa \mathrm{B}$ (Fig. 3A, $\mathrm{H}$ and $\mathrm{J}$ ). The MPO assay reflecting liver neutrophil activity (U/g) was reduced in the MH + IR compared with the NH + IR group or Ly $+\mathrm{MH}+\mathrm{IR}$ group (Fig. 5A). Similar changes were obtained for the effects of mild hypothermia pretreatment on IL-1 $\beta$, TNF- $\alpha$ and IL-6 (Fig. 5B-D).

\section{Discussion}

Organ damage by IR is associated with high morbidity and mortality. Accordingly, it is necessary to formulate treatment strategies for curing this disease. This has initiated numerous attempts to improve tissue protection against IR injury of the liver e.g., through ischemic preconditioning, pharmacological agents, hypothermia and so on. Mild hypothermia has achieved 
A

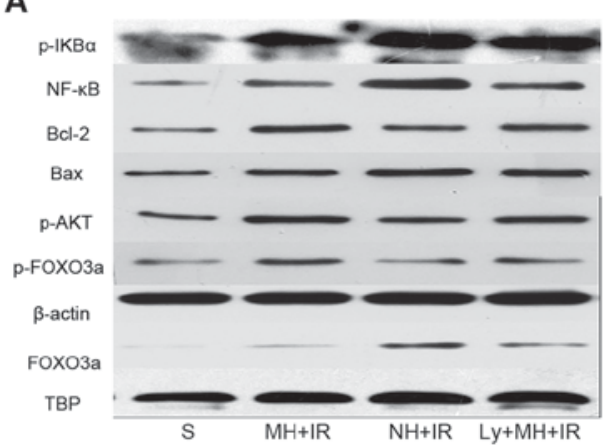

D

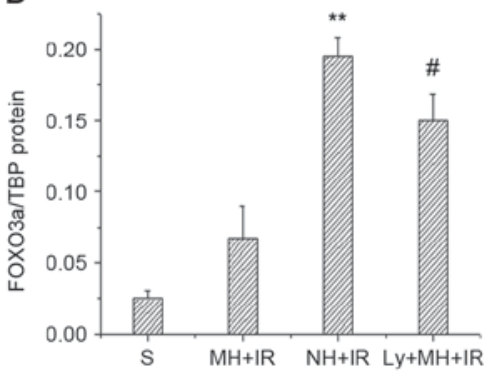

B

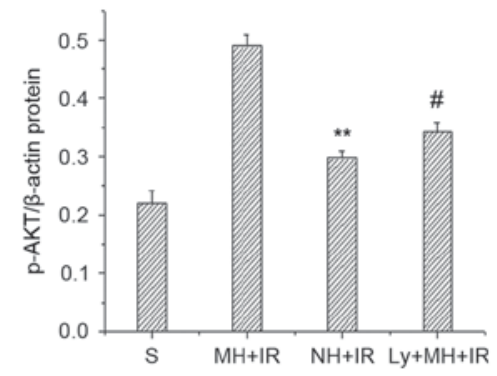

E

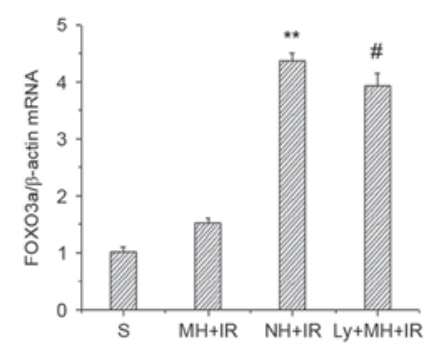

C

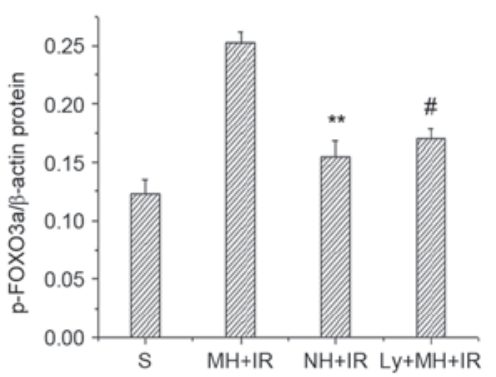

$\mathbf{F}$

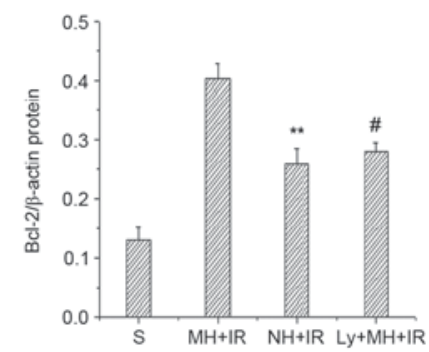

G

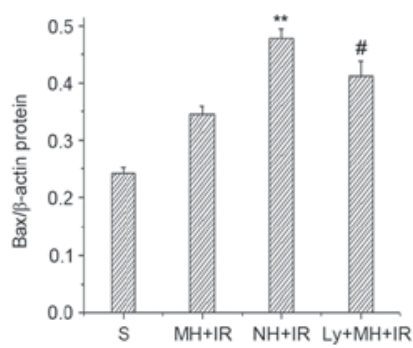

H

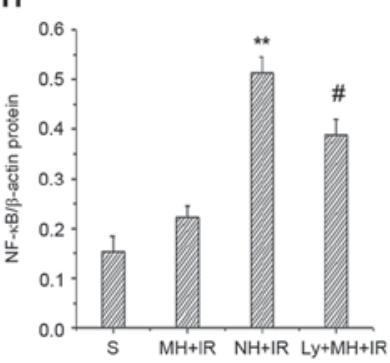

I

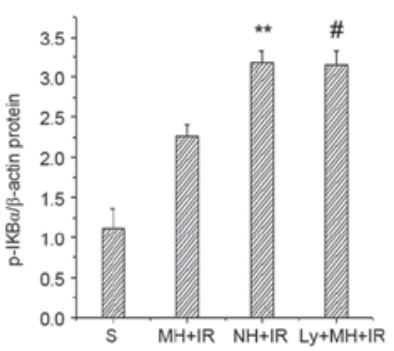

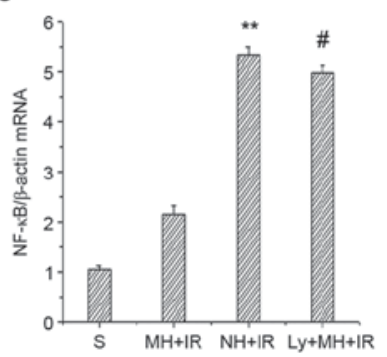

Figure 3. Mild hypothermia pretreatment activates phosphatidylinositol-3-kinase/AKT/FOXO3a signaling in liver IR injury. (A) Western blot analysis of p-AKT, p-FOXO3a, Bcl-2, Bax, p-IKB $\alpha$ and NF- $\mathrm{B}$; with $\beta$-actin as an internal control. Western blot analysis of nuclear FOXO3a and TBP served as an internal control. Band density of (B) p-AKT, (C) p-FOXO3a, (D) FOXO3a were measured, and p-AKT and p-FOXO3a were normalized to that of $\beta$-actin, FOXO3a was normalized to TBP. (E) RT-qPCR used to detect mRNA coding for FOXO3a. Data were normalized to $\beta$-actin gene expression. (F) Bcl-2,

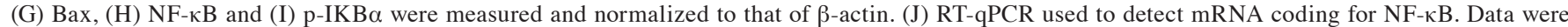
normalized to $\beta$-actin gene expression. Values are expressed as the mean \pm standard deviation ( $\mathrm{n}=8 /$ group). ${ }^{* *} \mathrm{P}<0.05 \mathrm{NH}+\mathrm{IR}$ group vs. MH $+\mathrm{IR}$ group, ${ }^{\#} \mathrm{P}<0.05 \mathrm{Ly}+\mathrm{MH}+\mathrm{IR}$ group vs. $\mathrm{MH}+\mathrm{IR}$ group. p-, phospho; IKB $\alpha, \mathrm{NF}-\kappa \mathrm{B}$ inhibitor $\alpha$; Bcl-2, Bcl-2 apoptosis regulator; Bax, Bcl-2 associated X apoptosis regulator; AKT, protein kinase B; FOXO3a, forkhead box O 3a; TBP, TATA-box binding protein; S, sham; MH, mild hypothermia pretreatment; IR, ischemia/reperfusion; NH, normothermic pretreatment; Ly, Ly294002.

A

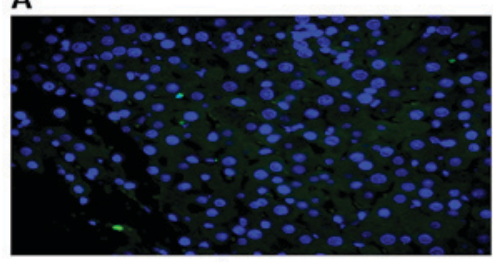

S

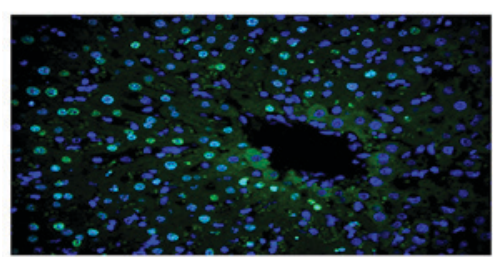

$\mathrm{NH}+\mathrm{IR}$

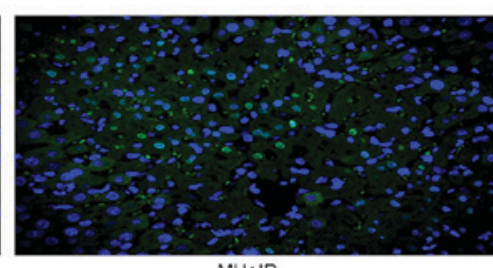

$\mathrm{MH}+\mathrm{IR}$

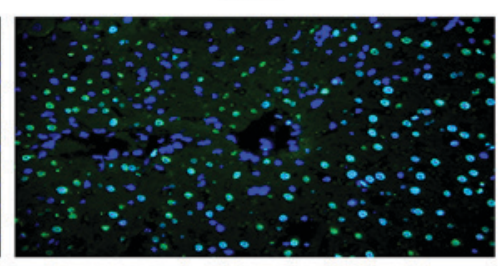

$L y+M H+I R$
B

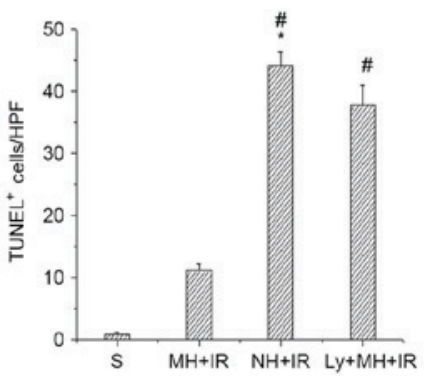

Figure 4. Mild hypothermia pretreatment promotes anti-apoptotic function and reduces hepatocellular apoptosis in liver IR injury. (A) TUNEL staining (magnification, x400). (B) The number of TUNEL-positive cells were counted from the liver section HPF. Values are expressed as the mean \pm standard deviation (n=8/group). ${ }^{*} \mathrm{P}<0.05$ vs. S group, ${ }^{*} \mathrm{P}<0.05$ vs. $\mathrm{MH}+\mathrm{IR}$ group. S, sham; MH, mild hypothermia pretreatment; IR, ischemia/reperfusion; $\mathrm{NH}$, normothermic pretreatment; Ly, Ly294002; TUNEL, terminal deoxynucleotidyl transferase dUTP nick-end labelling; HPF, high power field. 
A

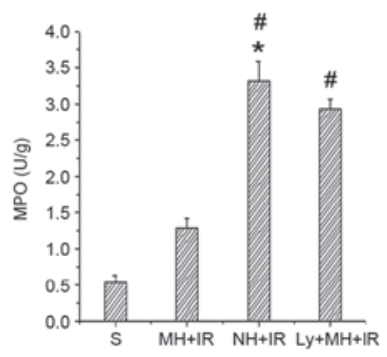

B

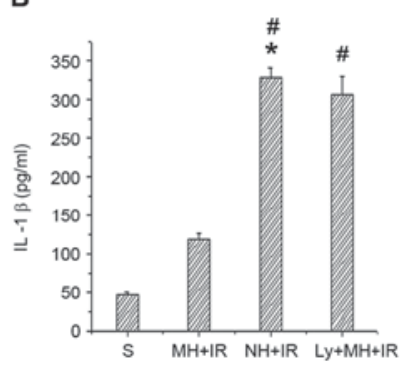

C

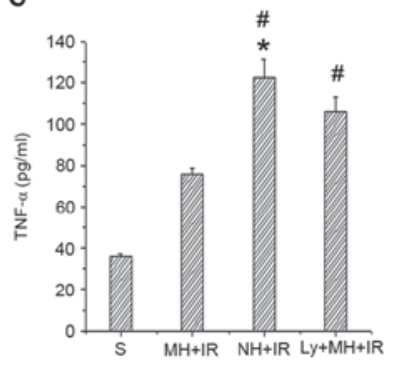

D

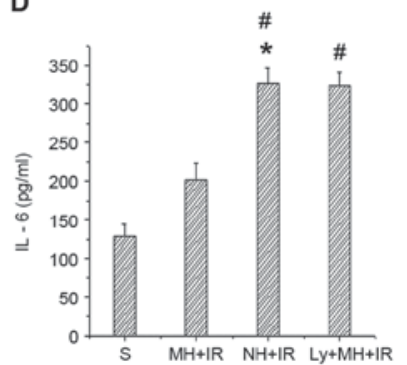

Figure 5. Mild hypothermia pretreatment inhibits the release of proinflammatory mediators in liver IR injury. (A) Neutrophil accumulation was determined by liver content of MPO activity (U/g). (B) IL-1 $\beta$ and (C) TNF- $\alpha$ and (D) IL- 6 were measured from serum by ELISA. Values are expressed as the mean \pm standard deviation ( $\mathrm{n}=8$ /group). ${ }^{*} \mathrm{P}<0.05$ vs. $\mathrm{S}$ group, ${ }^{\#} \mathrm{P}<0.05$ vs. $\mathrm{MH}+\mathrm{IR}$ group. MPO, myeloperoxidase; IL, interleukin; TNF- $\alpha$, tumor necrosis factor- $\alpha ; \mathrm{S}$, sham; MH, mild hypothermia pretreatment; IR, ischemia/reperfusion; NH, normothermic pretreatment; Ly, Ly294002.

good results in animal models of brain ischemic injury (21). In addition to improvements in brain ischemic injury, artificial hypothermia can significantly reduce ischemic damage of other organs, including the liver, kidney and myocardium $(22,23)$. Four large-scale prospective randomized controlled trials reported that mild hypothermia improved neural function in patients with cardiac arrest caused by ventricular fibrillation. The majority of previous studies $(8,9,24)$ have focused on investigation of therapeutic hypothermia $\left(32-34^{\circ} \mathrm{C}\right)$. However, in selective cases the application of mild hypothermia pretreatment may outweigh the risks, whereas fewer studies have been conducted using this method. The current in vivo study demonstrated that mild hypothermia pretreatment ameliorated liver IR and improved hepatocellular function, as evidenced by its ability to reduce sALT levels, histological liver damage, neutrophil activity and pro-inflammatory cytokine expression. A specific PI3K inhibitor (Ly294002) blocked the protective effect of mild hypothermia on liver IR. The present findings demonstrate a novel molecular protective mechanism of mild hypothermia pretreatment in rat livers subjected to $60 \mathrm{~min}$ warm ischemia and $6 \mathrm{~h}$ reperfusion via activation of the PI3K/AKT/FOXO3a signaling network.

FOXO3a is a member of the family of forkhead transcription factors, which have emerged to be critical regulators of gene expression, and to control oxidative stress and apoptosis in various cell types, and are regulated via phosphorylation by p-AKT (16). Without growth factor stimulation, FOXO3a is located in the nucleus and functions as a transcription factor. Once the PI3K signaling cascade is activated, FOXO3a is phosphorylated by $\mathrm{p}-\mathrm{AKT}$, reducing its DNA-binding capacity and exports p-FOXO3a from the nucleus to the cytoplasm, where it is degraded following ubiquitination (25). Previous studies $(26,27)$ have reported that PI3K/AKT pro-survival signaling is essential in heart and brain ischemic injury. IR causes inactivation of AKT, thereby decreasing phosphorylation of FOXO3a. However, once FOXO3a is phosphorylated, it is translocated into the cytoplasm, which downregulates the expression of pro-apoptosis molecules, such as Bax, and upregulates anti-apoptotic protein Bcl-2 $(28,29)$. The results of the current study demonstrated that IR insult downregulated the expression of p-AKT, whereas mild hypothermia pretreatment would block it. When compared with the $\mathrm{NH}+\mathrm{IR}$ and $\mathrm{Ly}+\mathrm{MH}+\mathrm{IR}$ groups, mild hypothermia pretreatment increased $\mathrm{p}-\mathrm{AKT}$ protein. The latter phosphorylated FOXO3a increased, resulting in the reduction of its DNA-binding capacity and export of p-FOXO3a from the nucleus to the cytoplasm, and decreased nucleus FOXO3a protein and mRNA, subsequently the expression of anti-apoptotic Bcl-2 was increased and pro-apoptotic Bax protein was deceased. There data was further confirmed by a TUNEL assay, which demonstrated that the number of apoptotic cells was increased in the $\mathrm{NH}+\mathrm{IR}$ group compared with the $\mathrm{MH}+\mathrm{IR}$ group ischemic liver samples. By contrast, ablation of PI3K/AKT signaling with Ly294002 depressed p-AKT, p-FOXO3a and Bcl-2; yet enhanced nuclear FOXO3a and Bax, and the number of apoptotic cells compared with the $\mathrm{MH}+\mathrm{IR}$ group. Thus, mild hypothermia pretreatment-mediated PI3K/AKT/FOXO3a signaling is essential to protect hepatocytes against IR-induced apoptotic.

FOXO3a signaling is known to induce proinflammatory cytokine gene expression, and it can regulate innate immune functions in respiratory epithelial cells (30). It is possible that mild hypothermia pretreatment attenuated inflammation response following IR injury via PI3K/AKT/FOXO3a signaling. Initially, liver IR triggers macrophage activation and neutrophil recruitment leading to aseptic inflammation (31). MPO assay is a measure of liver neutrophil activity. The results of the present study demonstrated that liver IR injury leads to increased MPO activity, whereas mild hypothermia pretreatment suppress MPO activity. However, blocking the effects of mild hypothermia with Ly294002 reversed the process. NF- $\mathrm{BB}$ is a ubiquitous inducible transcription factor, which induces target gene expression and promotes inflammatory responses (32). Numerous studies $(33,34)$ have reported strong evidence that NF- $\kappa$ B has a crucial role in tissue inflammatory responses during the pathogenesis of IR. NF- $\kappa \mathrm{B}$ knockout mice developed less ischemia damage following permanent focal insult (35). Increasing FOXO transcriptional activity enhances TNF- $\alpha$ and IL- $1 \beta$ production, whereas activation of $\mathrm{NF}-\kappa \mathrm{B}$ increases FOXO binding to the TNF- $\alpha$ and IL- $1 \beta$ promoters, indicating NF- $\kappa \mathrm{B}$ regulation of FOXO-mediated regulation of proinflammatory cytokines (36). In the current study, mild hypothermia pretreatment inhibited FOXO3a transcriptional activity, and ischemia resulted in increased $\mathrm{p}-\mathrm{IKB} \alpha$, which reduced the level of $\mathrm{NF}-\kappa \mathrm{B}$ and thus blocked activation of the $\mathrm{NF}-\kappa \mathrm{B}$ pathway that may attenuate inflammation though the regulation of proinflammatory cytokines (such as TNF- $\alpha$, IL-1 $\beta$ and IL-6). Furthermore, blocking PI3K/AKT with Ly294002 led to increased NF- $\kappa$ B transcription and TNF- $\alpha$, IL-1 $\beta$ and IL- 6 release, with similar results to the NH + IR group. The results demonstrated that inactivation 
of FOXO3a by mild hypothermia pretreatment can attenuate inflammation by regulation $\mathrm{NF}-\kappa \mathrm{B}$ activation and proinflammatory cytokine release.

In conclusion, the present study provided evidence that in vivo mild hypothermia pretreatment provides an efficient tool to protect the liver by inhibiting the degree of apoptosis and inflammation injury in the IR, leading to improved hepatic function, which may be due to activation of the $\mathrm{PI} 3 \mathrm{~K} / \mathrm{AKT} / \mathrm{FOXO3a}$ pathway. The study provides a rationale for novel therapeutic approaches for the management of hepatic injury triggered by IR. However, further studies are necessary to investigate the long-term effect of mild hypothermia pretreatment in liver IR injury. Subsequent clinical research needs to validate this conclusion.

\section{Acknowledgements}

This study was supported by National Natural Science Foundation of China (grant no. U1403222).

\section{References}

1. Selzner N, Rudiger H, Graf R and Clavien PA: Protective strategies against ischemic injury of the liver. Gastroenterology 125: 917-936, 2003.

2. Eltzschig HK and Eckle T: Ischemia and reperfusion-from mechanism to translation. Nat Med 17: 1391-1401, 2011.

3. Duarte S, Hamada T, Kuriyama N, Busuttil RW and Coito AJ: TIMP-1 deficiency leads to lethal partial hepatic ischemia and reperfusion injury. Hepatology 56: 1074-1085, 2012.

4. Jaeschke H and Woolbright BL: Current strategies to minimize hepatic ischemia-reperfusion injury by tageting reactive oxygen species. Transplant Rev (Orlando) 26: 103-114, 2012.

5. Bigelow WG, Callaghan JC and Hopps JA: General hypothermia for experimental intracardiac surgery. Ann Surg 132: 531-537, 1950

6. Miao YF, Wu H, Yang SF, Dai J, Qiu YM, Tao ZY and Zhang XH: 5 -adenosine monophosphate-induced hypothermia attenuates brain ischemia/reperfusion injury in a rat model by inhibiting the inflammatory response. Mediators Inflamm 2015: 520745, 2015.

7. Kida K, Shirozu K, Yu B, Mandeville JB, Bloch KD and Ichinose F: Beneficial effects of nitric oxide on outcomes after cardiac arrest and cardiopulmonary resuscitation in hypothermia-treated mice. Anesthesiology 120: 880-889, 2014.

8. Behrends M, Hirose R, Serkova NJ, Coatney JL, Bedolli M, Yardi J, Park YH and Niemann CU: Mild hypothermia reduces the inflammatory response and hepatic ischemia/reperfusion injury in rats. Liver Int 26: 734-741, 2006.

9. Niemann CU, Choi S, Behrends M, Hirose R, Noh J, Coatney JL, Roberts JP, Serkova NJ and Maher JJ: Mild hypothermia protects obese rats from fulminant hepatic necrosis induced by ischemia-reperfusion. Surgery 140: 404-412, 2006.

10. Zhang R, Zhang L, Manaenko A, Ye Z, Liu W and Sun X: Helium preconditioning protects mouse liver against ischemia and reperfusion injury through the PI3K/Akt pathway. J Hepatol 61: 1048-1055, 2014.

11. Suo L, Kang K, Wang X, Cao Y, Zhao H, Sun X, Tong L and Zhang F: Carvacrol alleviates ischemia reperfusion injury by regulating the PI3K-Akt pathway in rats. PLoS One 9: e104043, 2014.

12. Uranga RM, Katz S and Salvador GA: Enhanced phosphatidylinositol 3-kinase (PI3K)/Akt signaling has pleiotropic targets in hippocampal neurons exposed to iron-induced oxidative stress. J Biol Chem 288: 19773-19784, 2013.

13. Brunet A, Bonni A, Zigmond MJ, Lin MZ, Juo P, Hu LS, Anderson MJ, Arden KC, Blenis J and Greenberg ME: AKT promotes cells survival by phosphorylating and inhibiting a forkhead transcription factor. Cell 96: 857-868, 1999.

14. Lin K, Dorman JB, Rodan A and Kenyon C: daf-16: An HNF-3/forkhead family member that can function to double the life-span of Caenorhabditis elegans. Science 278: 1319-1322, 1997.

15. Furuyama T, Nakazawa T, Nakano I and Mori N: Identification of the differential distribution patterns of mRNAs and consensus binding sequences for mouse DAF-16 homologues. Biochem J 349: $629-634,2000$
16. Essers MA, de Vries-Smits LM, Barker N, Polderman PE, Burgering $\mathrm{BM}$ and Korswagen HC: Functional interaction between beta-catenin and FOXO in oxidative stress signaling. Science 308: 1181-1184, 2005.

17. Tzivion G, Dobson M and Ramakrishnan G: FoxO transcription factors; Regulation by AKT and 14-3-3 proteins. Biochim Biophys Acta 1813: 1938-1945, 2011.

18. Fischer AH, Jacobson KA, Rose J and Zeller R: Hematoxylin and eosin staining of tissue and cell sections. CSH Protoc 2008: pdb. prot4986, 2008.

19. Suzuki S, Toledo-Pereyra LH, Rodriguez FJ and Cejalvo D: Neutriphil infiltration as an important factor in liver ishcemia and reperfusion injury. Modulating effects of FK506 and cyclosporine. Transplantation 55: 1265-1272, 1993.

20. Livak KJ and Schmittgen TD: Analysis of relative gene expression data using real-time quantitative PCR and the 2(-Delta Delta C(T)) method. Methods 25: 402-408, 2001.

21. Tang Y, Liu X, Zhao J, Tan X, Liu B, Zhang G, Sun L, Han D, Chen $\mathrm{H}$ and Wang M: Hypothermia-induced ischemic tolerance is associated with Drp1 inhibition in cerebral ischemia-reperfusion injury of mice. Brain Res 1646: 73-83, 2016.

22. Niemann CU, Xu F, Choi S, Behrends M, Park Y, Hirose R and Maher JJ: Short passive cooling protects rats during hepatectomy by inducing heat shock proteins and limiting the induction of pro-inflammatory cytokines. J Surg Res 158: 43-52, 2010.

23. Niemann CU, Feiner J, Swain S, Bunting S, Friedman M, Crutchfield M, Broglio K, Hirose R, Roberts JP and Malinoski D: Therapeutic hypothermia in deceased organ donors and kidney-graft function. New Engl J Med 373: 405-414, 2015.

24. Bernard SA, Gray TW, Buist MD, Jones BM, Silvester W, Gutteridge G and Smith K: Treatment of comatose survivors of out-of-hospital cardiac arrest with induced hypothermia. New Engl J Med 346: 557-563, 2002.

25. Ma J, Ding Y, Fang X, Wang R and Sun Z: Protein kinase C- $\theta$ inhibits inducible regulatory $T$ cell differentiation via an AKT-Foxo1/3a-dependent pathway. J Immunol 188: 5337-5347, 2012.

26. Pei YH, Chen J, Xie L, Cai XM, Yang RH, Wang X and Gong JB: Hydroxytyrosol protects against myocardial ischemia/reperfusion injury through a PI3K/Akt-dependent mechanism. Mediators Inflamm 2016: 1232103, 2016.

27. Chen L, Wei X, Hou Y, Liu X, Li S, Sun B, Liu X and Liu H: Tetramethylpyrazine analogue $\mathrm{CXC} 195$ protects against cerebral ischemia/reperfusion-induced apoptosis through PI3K/Akt/GSK3 $\beta$ pathway in rats. Neurochem Int 66: 27-32, 2014.

28. Huang $\mathrm{C}, \mathrm{Gu} \mathrm{H}$, Zhang W, Herrmann JL and Wang M: Testosterone-down-regulated Akt pathway during cardiac ischemia/reperfusion: A mechanism involving BAD, Bcl-2 and FOXO3a. J Surg Res 164: e1-e11, 2010.

29. Luedde T, Kaplowitz N and Schwabe RF: Cell death and cell death responses in liver disease: Mechanisms and clinical relevance. Gastroenterology 147: 765-783.e4, 2014.

30. Seiler F, Hellberg J, Lepper PM, Kamyschnikow A, Herr C, Bischoff M, Langer F, Schäfers HJ, Lammert F, Menger MD, et al: FOXO transcription factors regulate innate immune mechanisms in respiratory epithelial cells. J Immunol 190: 1603-1613, 2013.

31. Zhai Y, Busuttil RW and Kupiec-Weglinski JW: Liver ischemia and reperfusion injury: New insights into mechanisms of innate adaptive immune-mediated tissue inflammation. Am J Transplant 11: 1563-1569, 2011.

32. Li Q and Verma IM: NF-kappaB regulation in the immune system. Nat Rev Immunol 2: 725-734, 2002.

33. Yang JC, Lin MW, Rau CS, Jeng SF, Lu TH, Wu YC, Chen YC, Tzeng SL, Wu CJ and Hsieh CH: Altered exosomal protein expression in the serum of NF- $\mathrm{kB}$ knockout mice following skeletal muscle ischemia-reperfusion injury. J Biomed Sci 22: 40, 2015.

34. Rao J, Qian X, Li G, Pan X, Zhang C, Zhang F, Zhai Y, Wang X and Lu L: ATF3-mediated NRF2/HO-1 signaling regulates TLR4 innate immune responses in mouse liver ischemia/reperfusion injury. Am J Transplant 15: 76-87, 2015.

35. Nurmi A, Lindsberg PJ, Koistinaho M, Zhang W, Juettler E, Karjalainen-Lindsberg ML, Weih F, Frank N, Schwaninger M and Koistinaho J: Nuclear factor-kappaB contributes to infarction after permanent focal ischemia. Stroke 35: 987-991, 2004.

36. Su D, Coudriet GM, Hyun Kim D, Lu Y, Perdomo G, Qu S, Slusher S, Tse HM, Piganelli J, Giannoukakis N, et al: FoxO1 links insulin resistance to proinflammatory cytokine IL-1beta production in macrophages. Diabetes 58: 2624-2633, 2009. 